\title{
Lesson Learned from the Emergence of Influenza Pandemic H1N1 in 2009 in Indonesia: The Importance of Influenza-Like Illness (ILI) Surveillance
}

\author{
Roselinda, ${ }^{1}$ Eka Pratiwi, ${ }^{1}$ Agustiningsih, ${ }^{1}$ and Vivi Setiawaty ${ }^{1,2}$ \\ ${ }^{1}$ Centre for Biomedical and Basic Technology of Health, NIHRD-MOH, Jl. Percetakan Negara No. 23, Central Jakarta 10560, Indonesia \\ ${ }^{2}$ Doctoral Program for Biomedical, Faculty of Medicine, University of Indonesia, Jl. Salemba Raya No. 4, \\ Central Jakarta 10430, Indonesia
}

Correspondence should be addressed to Vivi Setiawaty; vivisetiawaty@hotmail.com

Received 19 September 2013; Accepted 8 October 2013

Academic Editors: F. Ferrero and N. Uchide

Copyright (c) 2013 Roselinda et al. This is an open access article distributed under the Creative Commons Attribution License, which permits unrestricted use, distribution, and reproduction in any medium, provided the original work is properly cited.

\begin{abstract}
Background. In 2009 there were outbreaks of influenza pandemic H1N1 in Indonesia that were caused by different virus from the previous circulated H1N1. Further, the influenza-like illness (ILI) surveillance plays an important role in the early detection of influenza outbreaks in outpatients. To understand the disease burden of ILI in the community at the time of H1N1 pandemic 2009, a sentinel-based survey was performed. Methods. The nasal and throat swabs were obtained from 20 primary health centers of ILI sentinel in Indonesia in 2009. Identification of virus influenza pandemic H1N1 was carried out by real-time RT-PCR using primers that are specific for influenza A. Results. Out of 3254 ILI cases from community-based ILI surveillance in 2009, $11.03 \%$ cases were Influenza A positive and $42.59 \%$ cases were influenza pandemic H1N1. The first influenza pandemic HINI case was detected at week 15 in April, a case from the province of Banda Aceh, reaching a peak in August and ending at week 44 in November of 2009. Conclusion. The influenza pandemic H1N1 outbreak was detected in ILI surveillance network in Indonesia. This outbreak lasted for eight months which was the final wave of the influenza pandemic H1N1 in the world.
\end{abstract}

\section{Background}

Influenza is a disease that can potentially become a pandemic. From the history of the various subtypes that have been detected, there are several subtypes that caused pandemic such as H1N1 and H3N2. Influenza viruses circulate throughout the year in Indonesia, with seasonal activity often peaking during the rainy season (December-January) [1].

In 2009 there were outbreaks of H1N1pdm09 in the world. During the pandemic period, more than 214 countries and parts of the world reported positive laboratory confirmation of cases H1Nlpdm09 and recorded more than 2900 died in Europe [2]. In mid-April 2009, the first cases of 2009 pandemic influenza A ( $\mathrm{H} 1 \mathrm{Nl})$ were identified in the United States [3]. Viruses were detected in Italy in May 2009 on adult men who came from Mexico [4]. On July 2, 2009, South East
Asia had reported 1,866 cases [3]. Since then more than 27,000 confirmed cases and 260 fatalities [2, 4]. The H1N1pdm09 case among outpatient cases in Indonesia was first detected through influenza-like illness (ILI) surveillance in Indonesia on April 13, 2009 in boys aged 2 years old with a fever the day before, cough, and sore throat and domiciled in Banda Aceh.

The influenza A H1N1pdm09 virus originated from countries outside Indonesia that spread rapidly in the world. ILI surveillance is national influenza surveillance laboratories based on outpatient cases contained in each sentinel health center in 19 provinces in Indonesia. ILI surveillance in Indonesia plays an important role for the early detection of influenza H1N1pdm09 cases during outbreak. To have better estimation of the burden of ILI in the community at the time of the emergence of influenza H1N1pdm09 and observation on cases of potentially pandemic influenza in Indonesia, we 
conducted a sentinel-based survey in 20 sentinel sites by the relevant units in the Ministry of Health to assess the incidence of ILI caused by H1N1pdm09.

\section{Methods}

2.1. Data and Specimens Collection. ILI cases were obtained from patients with several symptoms including sudden fever with a temperature $\geq 37.8^{\circ} \mathrm{C}$ and with a cough, runny nose, sore throat, muscle pain, and shortness of breath that came to the outpatient at primary health center. Nose and throat swabs were obtained from outpatients in 20 health centers of ILI sentinel surveillance network in 19 provinces in Indonesia

Throat and nasal swabs from patients with ILI symptoms were homogenously collected using dacron swabs and obtained from January through December 2009. Swabs were placed into sterile hanks' balanced salt solution (HBSS) viral transport media (VTM) that contained gelatin, $100 \mathrm{U} / \mathrm{mL}$ penicillin, $100 \mu \mathrm{g} / \mathrm{mL}$ streptomycin, and $25 \mathrm{U} / \mathrm{mL}$ fungizone and then transported in cold condition to the regional reference laboratory. Specimens were sent every week to the Virology Laboratory of the CBBTH through the expedition using "one day service" to maintain the cold chain delivery of specimens.

2.2. Data Analysis. The data included in this paper underwent data extraction by trained study personnel and were organized by Microsoft Office Excel 2007. The linear regression test was used to determine the risk factor of infection and analyzed using Stata software version 09 (StataCorp).

2.3. Laboratory Diagnosis. The specimens were extracted using QIAmp viral mini kit (Qiagen, Hilden, Germany) according to the manufacturer's instruction. Real-time Reverse Transcriptase-Polimerase Chain Reaction (RT-PCR) was performed according to a recommended protocol from the United States Centers for Disease Control and Prevention (US CDC) on an IQ5 Bio-Rad real-time PCR instrument (Bio-Rad, US) $[1,5]$. RT-PCR was preformed using primers and probes that are specific for influenza $A$, influenza $B$, H1N1, H3N2, H5N1, and H1N1pdm09. Primers and probes were provided by the Center for Disease Control and Prevention, USA. All laboratory tests were performed at the Virology Laboratory Center for Biomedical and Basic Technology of Health (CBBTH), Jakarta.

Each case of ILI was laboratory-confirmed as negative for influenza A or positive for pandemic influenza A (H1N1)2009, influenza A (H3N2) or unsubtypable influenza A, as appropriate according to the recommended protocol from US CDC. PCR results of influenza were sent to FluNet for regular report and feedback to the sentinel and programs.

\section{Results}

A total of 3254 ILI cases $(n=1595$ females $(50.9 \%)$ and $n=$ 1659 males $(49.1 \%)$ ) were obtained from the outpatient case in the health centers of ILI sentinel surveillance. From the results of the real-time RT-PCR, positive cases of influenza
A were 359 cases (11.03\%) and positive cases for H1N1pdm09 were 187 cases (52.08\%). Influenza A unsubtypeable were 89 cases $(24.79 \%)$. Influenza A (H3N2) and H1N1 human were 41 cases $(11.42 \%)$ and 42 cases (11.69\%), respectively. Table 1 showed that the highest ILI cases were 1311 cases $(40.29 \%)$ at the age group 6-14 years, followed by age group $0-5$ years with 908 cases $(27.1 \%)$, whereas most cases of influenza A were also in the same age group in the amount of 186 cases (14.19\%) and followed by the age group 15-24 years for 62 cases $(17.51 \%)$.

Based on ILI surveillance in 2009, H1N1pdm09 case was firstly detected on April 2009. The number of patients with ILI symptoms increased since April and in July to August there were four times ILI cases reported than that in January to March. The number of specimens collected was also increasing. Most of the ILI cases were detected as H1N1pdm09. The H1N1pdm09 cases also reaching a peak at $71.5 \%$ in July to August with 176 influenza H1N1pdm09 were reported and decreased in September 2009. In 2009, H1N1pdm09 became a dominant subtype of flu A. Almost $50 \%$ of flu A circulating in 2009 was H1N1pdm09 (187 H1N1pdm09 out of 359 flu A in total). Although there were an H1N1pdm09 outbreak, the low number of seasonal influenza A (H1), A (H3) is still found and the flu B cases remain stable. The number of ILI and influenza subtypes from January to December 2009 was shown in Table 2.

The first H1N1pdm09 case was reported from Banda Raya health center in the Nanggroe Aceh Darussalam province in April 2009. The specimen was collected on April 13, 2009 but confirmed influenza H1N1pdm09 in June 2009, after we received new primers and probe of influenza H1N1pdm09 in early May 2009 from US CDC, the World Health Organization Collaborating Centre (WHO CC) for influenza. In 2009, Banda Raya health center reported and collected specimens from 117 ILI cases in which 20 cases were reported as influenza positive and only 3 out of 20 influenza positive were H1N1pdm09 (Table 3). In 2009 not all ILI sentinel sites reported H1N1pdm09. There were only 12 out of 20 health centres of ILI sentinels reported patients infected with H1N1pdm09 (Table 3). Figure 1 shows the distribution of the ILI sentinel sites that reported the H1N1pdm09 throughout Indonesia.

Overall, 13.5\% (439/3254) of ILI cases throughout 2009 were positive for influenza viruses with influenza A virus and influenza B virus were $81.8 \%$ (359) and $18.2 \%$ (80) cases, respectively.

Figure 2 shows that the first case of H1N1pdm09 appeared at week 15 and reached a peak at weeks 28 to 30 and decreases slowly and ends at week 34 . The virus then was detected again at week 41 to 44 in November. Until the end of 2009, the virus never appeared again.

Risk factor analysis of influenza H1N1pdm09 infection among samples was described in Table 4. Proportion of male infected with influenza H1N1pdm09 is higher than female, 112 out of 190 (58.95\%). The age group 15-24 y.o had higher risk to be infected by influenza H1N1pdm09 among other age groups. There were 47 out of 65 cases $(75.81 \%)$ infected by influenza H1N1pdm09 from the age group 15-24 y.o. The risk to be infected by influenza H1N1pdm09 increased 6,7 times 
TABLE 1: Characteristics of influenza A cases from ILI surveillance in 2009 in Indonesia.

\begin{tabular}{|c|c|c|c|}
\hline & ILI Total cases & Flu A negative & Flu A positive \\
\hline Outpatients & 3254 & $2895(88.97)$ & $359(11.03)$ \\
\hline Flu A (H1N1pdm09) & & & $187(52.08)$ \\
\hline Flu A (H3N2) & & & $41(11.42)$ \\
\hline Flu A (H1N1human) & & & $42(11.69)$ \\
\hline Flu A unsubtypeable & & & 89 (24.79) \\
\hline \multicolumn{4}{|l|}{ Gender } \\
\hline Male & $1659(50.98)$ & $1469(88.55)$ & $190(11.45)$ \\
\hline Female & 1595 (49.02) & $1426(89.40)$ & $169(10.60)$ \\
\hline \multicolumn{4}{|l|}{ Age } \\
\hline $0-5$ y.o. ${ }^{*}$ & $908(27.90)$ & $850(93.61)$ & $58(6.39)$ \\
\hline 6-14 у.о.* & $1311(40.29)$ & $1125(85.81)$ & $186(14.19)$ \\
\hline $15-24$ y.o. $^{*}$ & $354(10.88)$ & $292(82.49)$ & $62(17.51)$ \\
\hline$>25$ y.o. ${ }^{*}$ & $681(20.93)$ & $628(92.22)$ & $53(7.78)$ \\
\hline
\end{tabular}

*Y.o: years old.

TABLE 2: Case proportion, type, and subtype of seasonal influenza monthly.

\begin{tabular}{|c|c|c|c|c|c|c|c|}
\hline Month & No. of ILI cases & Flu B & H1N1 & $\mathrm{H} 3 \mathrm{~N} 2$ & $\mathrm{H} 5 \mathrm{~N} 1$ & H1pdm09 & Flu A not type \\
\hline January & 87 & 10 & 3 & 5 & 0 & 0 & 8 \\
\hline February & 81 & 14 & 4 & 1 & 0 & 0 & 2 \\
\hline March & 103 & 18 & 6 & 0 & 0 & 0 & 2 \\
\hline April & 277 & 9 & 4 & 2 & 0 & 1 & 2 \\
\hline May & 345 & 8 & 4 & 2 & 0 & 0 & 0 \\
\hline June & 366 & 1 & 9 & 10 & 0 & 8 & 2 \\
\hline July & 474 & 0 & 10 & 9 & 0 & 83 & 16 \\
\hline August & 475 & 0 & 1 & 6 & 0 & 93 & 28 \\
\hline September & 212 & 0 & 0 & 3 & 0 & 1 & 12 \\
\hline October & 279 & 9 & 0 & 1 & 0 & 0 & 11 \\
\hline November & 277 & 4 & 0 & 1 & 0 & 1 & 3 \\
\hline December & 278 & 7 & 1 & 1 & 0 & 0 & 4 \\
\hline Total & 3254 & 80 & 42 & 41 & 0 & 187 & 89 \\
\hline
\end{tabular}

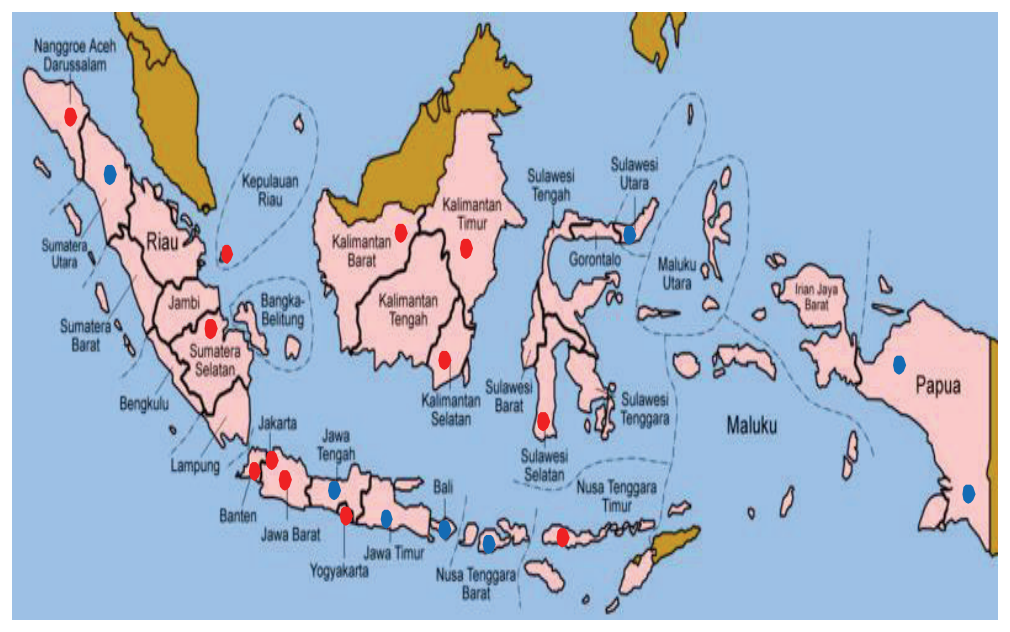

H1N1 pdm has been found

- H1N1 pdm has not been found

FIGURE 1: Map of ILI sentinel sites and provinces in Indonesia. 
TABLE 3: Number of ILI cases, type, and subtype of influenza per sentinel sites.

\begin{tabular}{|c|c|c|c|c|c|c|}
\hline Sentinel sites (province) & ILI & Flu B & H1N1 & H3N2 & H1N1pdm09 & Flu A not type \\
\hline 7 ULU (South Sumatra) & 204 & 0 & 0 & 0 & 15 & 34 \\
\hline Banda Raya (Nanggroe Aceh Darussalam) & 117 & 5 & 0 & 3 & 3 & 8 \\
\hline Batu Aji (Kepulauan Riau) & 242 & 0 & 1 & 7 & 4 & 19 \\
\hline Curug (Banten) & 196 & 7 & 9 & 2 & 33 & 51 \\
\hline Dinoyo (East Java) & 219 & 0 & 0 & 0 & 0 & 1 \\
\hline Jayapura Utara (Papua) & 68 & 0 & 0 & 0 & 0 & 12 \\
\hline Karang Taliwang (West Nusa Tenggara) & 308 & 0 & 1 & 4 & 0 & 5 \\
\hline Klandasan Ilir (East Kalimantan) & 73 & 1 & 0 & 0 & 12 & 13 \\
\hline Kota Gede (Yogyakarta) & 138 & 0 & 1 & 0 & 9 & 10 \\
\hline Mopah (Papua) & 149 & 10 & 1 & 1 & 0 & 9 \\
\hline Padasuka (West Java) & 95 & 4 & 1 & 0 & 25 & 29 \\
\hline Pandanaran (Central Java) & 166 & 0 & 0 & 0 & 0 & 0 \\
\hline Pekauman (South Kalimantan) & 192 & 9 & 7 & 9 & 26 & 50 \\
\hline Denpasar Selatan (Bali) & 200 & 0 & 0 & 2 & 0 & 2 \\
\hline Siantan Ilir (West Kalimantan) & 178 & 1 & 6 & 2 & 30 & 34 \\
\hline Sikumana (East Nusa Tenggara) & 151 & 2 & 2 & 3 & 7 & 6 \\
\hline Sudiang (South Sulawesi) & 256 & 0 & 2 & 0 & 1 & 37 \\
\hline Teladan (North Sumatra) & 121 & 2 & 0 & 2 & 0 & 17 \\
\hline Tikala baru (North Sulawesi) & 75 & 16 & 2 & 3 & 0 & 10 \\
\hline Utan Kayu Utara (Jakarta) & 195 & 0 & 0 & 0 & 22 & 2 \\
\hline Total & 3254 & 80 & 42 & 41 & 187 & 89 \\
\hline
\end{tabular}

TABLE 4: Risk factor of influenza H1N1pdm09 infection.

\begin{tabular}{lcccc}
\hline & \multirow{2}{*}{ Flu A $(n=359)$} & Flu A & & OR (95\% CI) \\
\hline $\begin{array}{l}\text { Gender } \\
\text { Male }\end{array}$ & 190 & $78(41.05)$ & $112(58.95)$ & Reference group \\
Female & 169 & $94(55.62)$ & $75(44.38)$ & $0.58(0.37-0.90)$ \\
Age group & & & & 0.016 \\
0-4 y.o & 58 & $40(68.97)$ & $18(31.03)$ & Reference group \\
$5-14$ y.o & 186 & $84(45.16)$ & $102(54.84)$ & $2.84(1.50-5.35)$ \\
$15-24$ y.o & 62 & $15(24.19)$ & $187(75.81)$ & $6.70(2.98-15.07)$ \\
$\geq 25$ y.o & 53 & $33(62.26)$ & & 0.016 \\
\end{tabular}

(OR: 2.98-15.07, with $P$ value 0.001$)$ in this age group than age group 0-4 y.o based on statistical analysis (Table 4).

\section{Discussions}

Influenza viruses by nature are unstable and therefore the occurrence of the next influenza pandemic remains unpredictable. Early detection of pandemic influenza at national level is a public health concern. Special concern needs to be addressed to Indonesia as a tropical country where influenza viruses are circulating throughout the year without well-defined pattern. In Indonesia, influenza surveillance is the essential system for monitoring routinely the influenza activity especially when the influenza cases with pandemic potential occurred [6].

After the WHO announced the two first cases of confirmed influenza H1N1pdm09 on week 16 in 2009, the virus rapidly spread throughout the world. The specimens of these two cases were collected on March 30 and April 1 and confirmed on April 15 and 17, respectively [7]. The US CDC as a WHO CC for influenza shared the primers and probes to detect the influenza H1N1pdm09 to National influenza Centres (NIC) including Indonesia NIC who conduct the influenza surveillance. As a NIC, we collect, identify, analyze, and also isolate influenza strain from the clinical specimens.

When pandemic occurred in April 2009, there were some unsubtypeable flu A found in ILI samples in Indonesia (Table 3). Following the distribution of primers and probes for influenza H1N1pdm09 detection from US CDC that we received on May 2009, we retested the unsubtype flu A. We found that one of the case reported ILI symptoms with specimens collected on April 13, 2009 was confirmed positive of influenza H1N1pdm09. That was the only influenza H1N1pdm09 case found on April. On May, we did not find any 


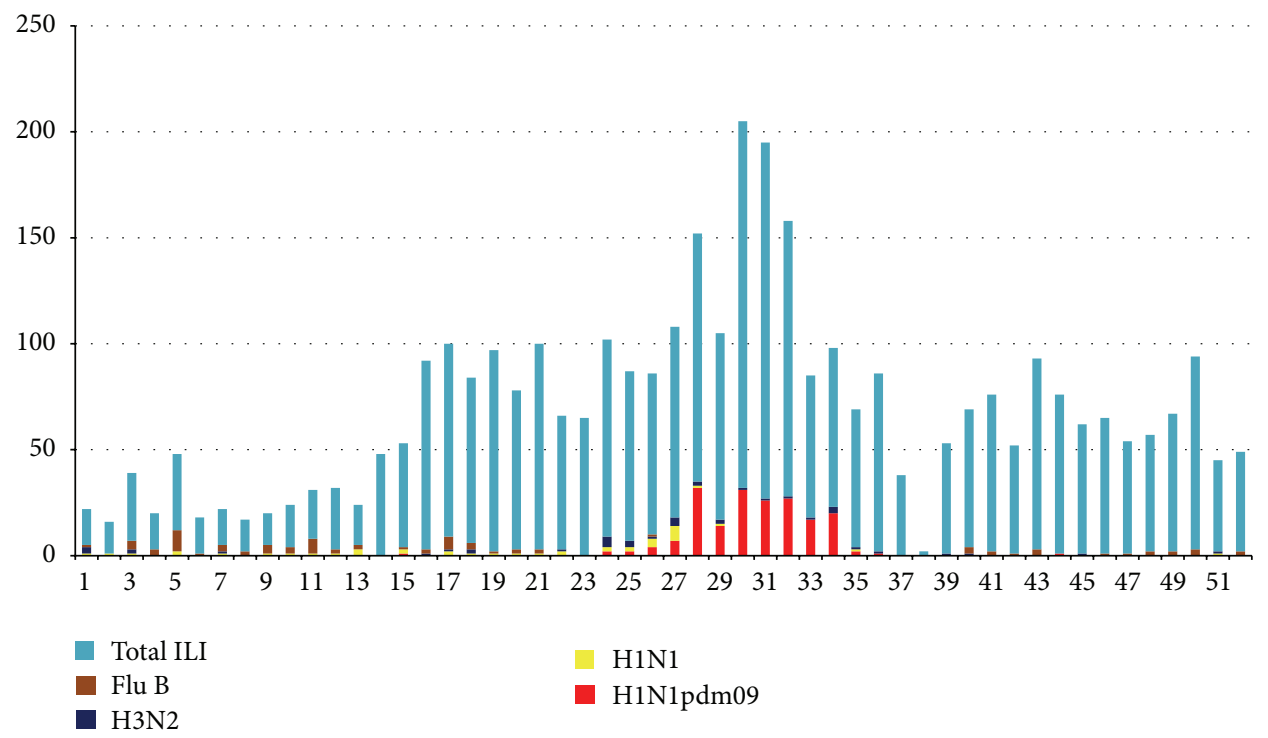

Figure 2: Graph distribution of ILI cases in Indonesia with seasonal influenza weekly 2009.

cases of influenza H1N1pdm09 but from June to August the influenza H1N1pdm09 cases increased rapidly.

WHO with the Global influenza Surveillance Network (GISN) has monitored influenza activity for more than 60 years [8]. Indonesia as WHO member state is included in the network with the routine influenza surveillance activity for 14 years since 1999. As part of the network, we should provide data of influenza activity to WHO. There are some ways to provide the data and one of them is sharing data through FluNet, a web-based electronic data for reporting the influenza activity. Unfortunately, Indonesia did not share the influenza data through FluNet until 2010. Therefore, the influenza data in 2009 especially for influenza pandemic activity from ILI surveillance were not recorded globally in WHO system. This situation occurred not only in Indonesia as WHO reported that only $54 \%$ WHO state members reported the activity for influenza pandemic detection [8].

The growth of the international trade and travel across the world increases the risk of emergence pathogen including pandemic influenza. The international travel can enhance the spread of the influenza H1N1pdm09 [8]. From the 20 sentinels of ILI surveillance, there were only 12 sentinels reporting influenza H1N1pdm09 from April to December 2009. The characteristics of the 12 health centers are almost the same where they are in the city of provincial capital with lots of travelers. Especially, in Banda Aceh, there were a lot of foreign volunteers for short visit who wanted to remedy Aceh people after the tsunami in early 2005.

This study describes the influenza activity during H1N1 pandemic in 2009 where H1N1pdm09 cases were mostly found in young adult (15-24 years). Based on risk factor analysis, age group 15-24 years old has higher risk to be infected by H1N1pdm09. This finding is consistent with the previous study that has been conducted in 11 states where $75 \%$ of confirmed cases of influenza H1N1pdm09 infection were at age $<30$ years with a peak at 10-19 years of age [9].
The cross-reactive antibodies to the pandemic virus were detected frequently in people aged $>60$ years old than in younger adults and children. This supports the theory that people at a young age do not have immunity to antigenically distinct influenza viruses $[10,11]$. The highest percentage of H1N1pdm09 cases found in Indonesia was in the end of July 2009 (week 28), indicating the rapid spread of this virus widely since the first occurrence in April 2009 in US. However, influenza B, H1N1, and $\mathrm{H} 3 \mathrm{~N} 2$ viruses also remained detected during pandemic as seen in other studies in US [12].

Regarding the occurrence of influenza H1N1pdm09 in 2009 , some countries impose early mitigation effort to avoid events that involve a lot of people. Unfortunately, in Indonesia early mitigation can only be carried out in a limited way as there was limitation in laboratory testing for early detection due to the availability of primers and probes for laboratory testing. Monitoring of the increasing number of cases of H1N1pdm09 and fatal cases caused by H1N1pdm09 virus was the only effort during outbreaks in 2009. There were no special precautions at the health centres who reported confirmed cases of influenza H1N1pdm09. This is in accordance with the WHO recommendation for close monitoring; however, the recommendation does not control the viral spreads in relation to international travel [13].

\section{Conclusions}

Influenza A virus infection (H1N1pdm09) as the cause of a pandemic in 2009 worldwide was also detected in Indonesia through sentinels in ILI surveillance. Since the spread of H1N1pdm09 virus, the number of ILI cases increased and H1N1pdm09 became the dominant influenza virus subtypes throughout the 2009. The risk factor of new subtype of influenza A H1N1pdm09 infection increased in male and young adults. The detection of new virus H1N1pdm09 through surveillance activity shows that ILI surveillance is 
very important as a sustain activity. ILI surveillance has crucial roles especially in the monitoring of the patterns of the influenza virus circulated in Indonesia and for early detection of the emergence novel influenza viruses.

\section{Acknowledgments}

The authors thank Director General for National Institute of Health Research and Development, Dr. Trihono, US CDC, WHO, hospitals, primary health centres, district and provincial health office, and laboratory team for their support and good collaboration. This study was funded through US CDC Grant no. 5U50/024413-05 for influenza surveillance. The authors declare that there is no conflict of interests.

\section{References}

[1] H. Kosasih, R. Nurhayati, A. Klimov, X. Xiyan, S. Lindstrom et al., "Surveillance of Influenza in Indonesia, 2003-2007," Influenza and Other Respiratory Viruses, vol. 7, no. 3, pp. 312320, 2013.

[2] European Centre for Disease Prevention and Control (ECDC), "Announced cumulative number of confirmed fatal cases of 2009 pandemic influenza A(H1N1) in EU and EFTA (Updated 3 May 2010). Stockholm:ECDC," http://ecdc.europa.eu/en/ healthtopics/H1N1/epidemiological_data/Pages/number_confirmed_fatal_2009_pandemic_influenza_cases.aspx.

[3] World Health Organization (WHO), "Influenza-like illness in the United States and Mexico. Geneva, Switzerland, WHO," 2009, http://www.who.int/csr/don/2009_04_24/en/index.html.

[4] C. Rizzo, M. C. Rota, A. Bella et al., "Response to the 2009 influenza A (H1N1) pandemic in Italy," Eurosurveillance, vol. 15, no. 49, p. $6,2010$.

[5] World Health Organization (WHO), "CDC protocol of realtime RTPCR for influenza A(H1N1). Geneva, Switzerland, WHO, revision 2," 2009, http://www.who.int/entity/csr/resources/ swineflu/CDCRealtimeRTPCR_SwineH1Assay-2009_20090430.pdf.

[6] B. K. Singh, N. J. Savill, N. M. Ferguson, C. Robertson, and M. E. Woolhouse, "Rapid detection of pandemic influenza in the presence of seasonal influenza," BMC Public Health, vol. 10, article 726, 2010.

[7] Center for Disease Control and Prevention, "Swine Influenza A, (H1N1) infection in two children-southern California, MarchApril 2009," Morbidity and Mortality Weekly Report, vol. 58, pp. 400-402, 2009.

[8] S. Briand, A. Mounts, and M. Chamberland, "Challenges of global surveillance during an influenza pandemic," Public Health, vol. 125, no. 5, pp. 247-256, 2011.

[9] M. Khanna, L. Saxena, A. Gupta, B. Kumar, and R. Rajput, "Influenza pandemics of 1918 and 2009: a comparative account," Future Virology, vol. 8, no. 4, pp. 1-8, 2013.

[10] G. Chowell, S. Echevarría-Zuno, C. Viboud et al., "Recrudescent wave of pandemic A/H1N1 influenza in Mexico, winter 20112012: age shift and severity," PLOS Currents, vol. 4, Article ID RRN1306, 2012.

[11] T. Reichert, G. Chowell, H. Nishiura, R. A. Christensen, and J. A. McCullers, "Does Glycosylation as a modifier of Original Antigenic Sin explain the case age distribution and unusual toxicity in pandemic novel H1N1 influenza?" BMC Infectious Diseases, vol. 10, article 5, 2010.
[12] L. Brammer, L. Blanton, S. Epperson et al., "Surveillance for influenza during the 2009 influenza a (H1N1) pandemic-United States, April 2009-March 2010," Clinical Infectious Diseases, vol. 52, no. 1, pp. S27-S35, 2011.

[13] World Health Organization, "New Influenza A(H1N1) virus infections: global surveillance summary," Weekly Epidemiological Record, vol. 84, pp. 173-184, 2009. 


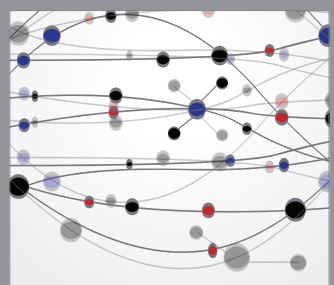

The Scientific World Journal
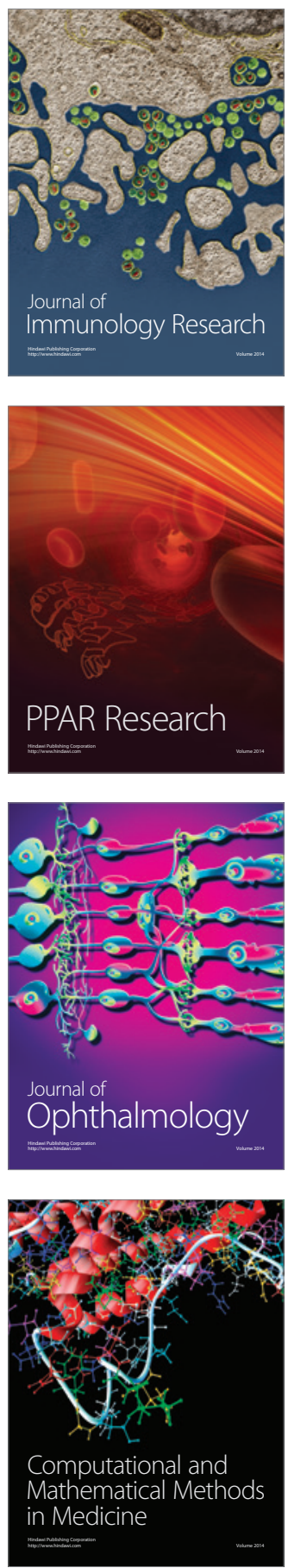

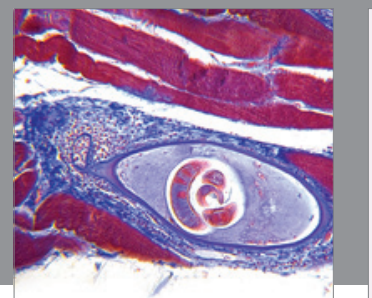

Gastroenterology

Research and Practice
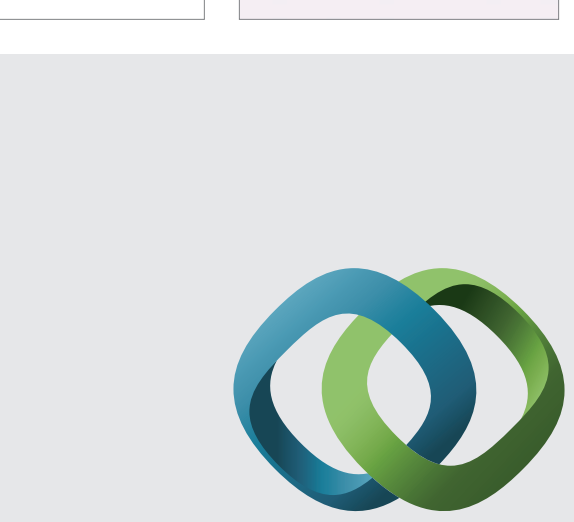

\section{Hindawi}

Submit your manuscripts at

http://www.hindawi.com
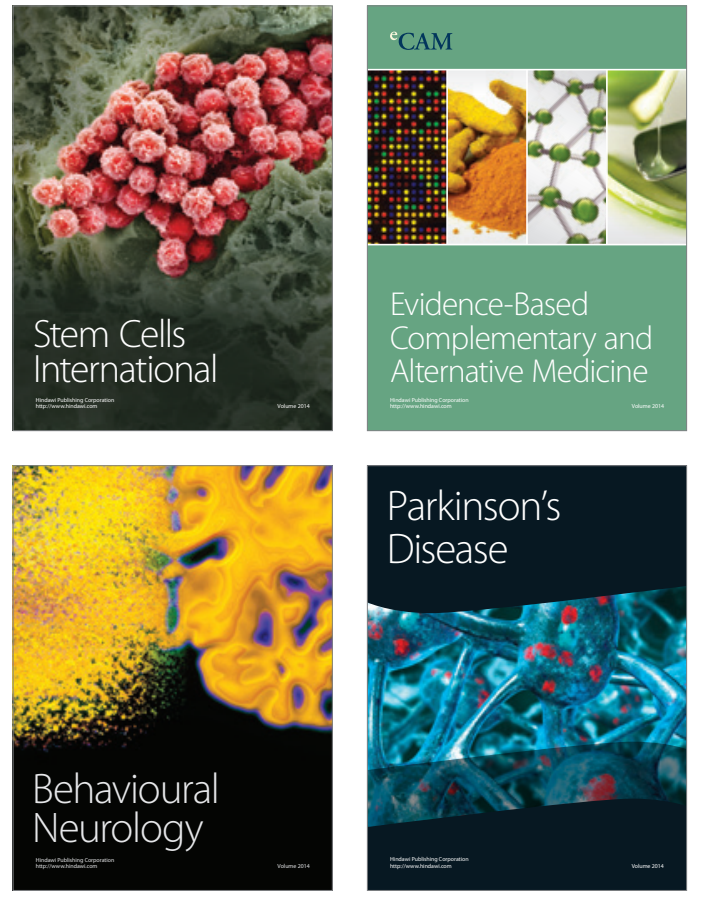
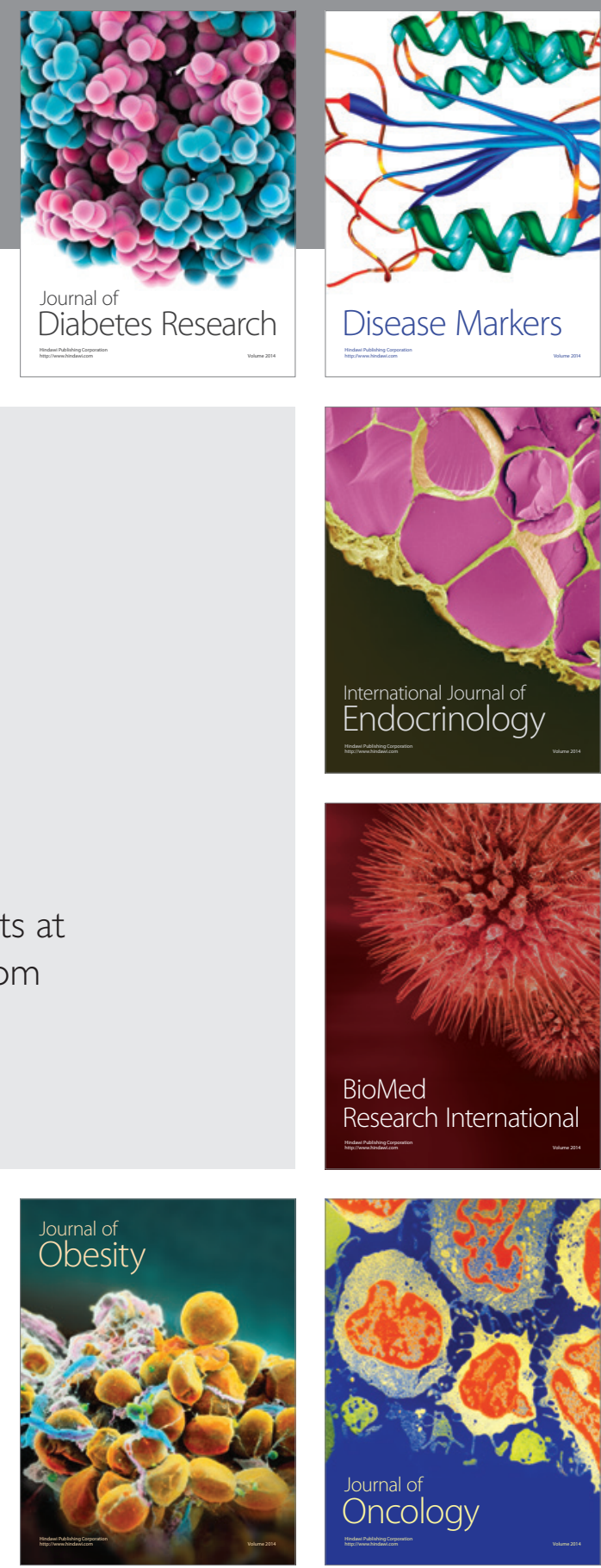

Disease Markers
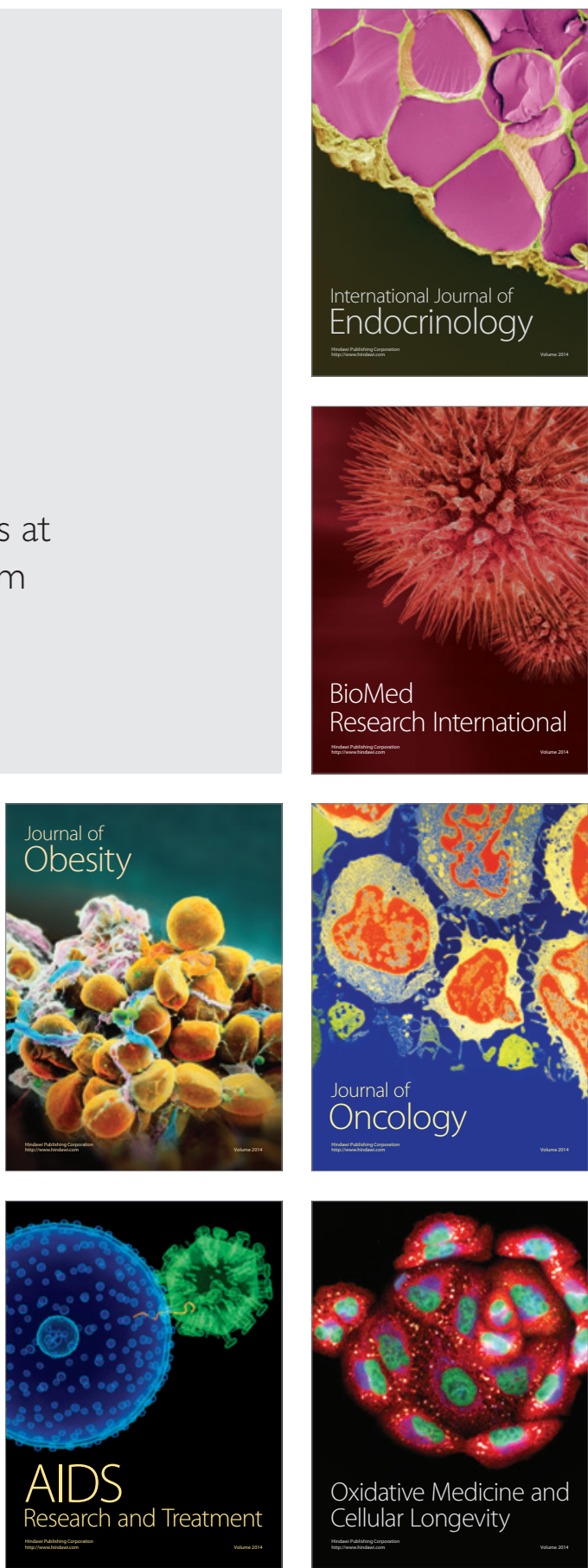